partial that means podoplanin is one of the involved mechanisms but others have to be identified.

Disclosure of interest None declared

\section{P068 INCREASE OF AEROBIC GLYCOLYSIS MEDIATED BY ACTIVATED T HELPER CELLS DRIVES SYNOVIAL FIBROBLASTS TOWARDS AN INFLAMMATORY PHENOTYPE}

\begin{abstract}
${ }^{1} \mathrm{P}$ Kvacskay ${ }^{*},{ }^{1} \mathrm{M}$ Souto-Carneiro, ${ }^{2} \mathrm{R}$ de Albuquerque Carvalho, ${ }^{1} \mathrm{~J}-\mathrm{H}$ Schnotz, ${ }^{1} \mathrm{~S}$ Krienke, ${ }^{3} \mathrm{KD}$ Klika, ${ }^{1} \mathrm{~T}$ Tretter, ${ }^{1} \mathrm{H}-\mathrm{M}$ Lorenz, ${ }^{1} \mathrm{~L}-\mathrm{O}$ Tykocinski. ${ }^{1}$ Internal Medicine $\mathrm{V}$ - Division of Rheumatology, University Hospital Heidelberg, Heidelberg, Germany; ${ }^{2}$ NMR Centre, Department of Biochemistry, University of Coimbra, Coimbra, Portugal; ${ }^{3}$ Molecular Structure Analysis, German Cancer Research Centre (DKFZ), Heidelberg, Germany
\end{abstract}

\subsection{6/annrheumdis-2018-EWRR2018.86}

Introduction There is growing evidence for a dysregulated glucose metabolism of synovial fibroblasts (SF) in rheumatoid arthritis (RA) being a prerequisite for their aggressive phenotype. As yet, little is known about the influence of immune cells on the metabolism of SF although local infiltration of leucocytes constitutes a hallmark in the pathogenesis of RA.

Objectives In this study, we investigated the effect of $T$ helper $\left(\mathrm{T}_{\mathrm{h}}\right)$ cells on the glucose metabolism and cytokine production of non-inflammatory and inflammatory SF in vitro.

Methods RASF as well as SF from patients with osteoarthritis (OA) were cultured in the presence of a stable glucose isotope $\left(\mathrm{U}^{13} \mathrm{C}\right)$ and stimulated with culture supernatants $(\mathrm{SN})$ of activated $\mathrm{T}_{\mathrm{h}}$ cells. Lactate production was measured by proton nuclear magnetic resonance spectroscopy (H-NMR). Secretion of interleukin (IL)-6, IL-8 and matrix metalloprotease (MMP)-3 was quantified by ELISA. The expression of hexokinase (HK)-II, pyruvate kinase (PK)-M2 and pyruvate dehydrogenase (PDH) was analysed by $\mathrm{PCR}$, western blot and immunofluorescence. Janus kinases (JAKs) were blocked by Baricitinib or Tofacitinib, glycolysis was inhibited by 3-Bromopyruvate (3 BP) or Fx11.

Results In the absence of stimulation, RASF showed a significantly higher lactate production and IL-6 and MMP-3 secretion compared to OASF. Stimulation by $\mathrm{T}_{\mathrm{h}}$ cell $\mathrm{SN}$ strikingly changed the metabolic profile of both RASF and OASF by inducing a shift towards aerobic glycolysis with strongly increased lactate production. In parallel, a significant increase in IL-6 and MMP-3 secretion was induced. Interestingly, Bariticinib and Tofacitinib as well as glycolytic inhibitors significantly reduced both the production of lactate and the secretion of inflammatory cytokines. Finally, perpetual stimulation of non-inflammatory OASF by $T_{h}$ cell $S N$ triggered an inflammatory phenotype characterised by significantly higher amounts of lactate, IL-6 and MMP-3 compared to non- or single stimulated SF.

Conclusions Chronic stimulation of non-inflammatory SF by activated $T_{h}$ cells provoked an aggressive phenotype with strongly increased glycolytic activity and upregulated secretion of inflammatory cytokines. This was blocked by both JAKand glycolytic inhibitors. These observations suggest that the $\mathrm{T}_{\mathrm{h}}$ cell-mediated metabolic switch towards aerobic glycolysis in SF is an important step in the pathogenesis of RA. Targeting this mechanism could provide a new strategy in the therapy of RA.

Disclosure of interest None declared

\section{P069 S100A9 MEDIATES ACUTE NOCICEPTIVE PAIN IN EXPERIMENTAL SYNOVITIS}

A Blom, M van den Bosch, E Geven, E Blaney Davidson, P van der Kraan, P van Lent*. Experimental Rheumatology, Radboud University Medical Centre, Nijmegen, Netherlands

\subsection{6/annrheumdis-2018-EWRR2018.87}

Introduction Synovitis-associated pain is an important aspect of arthritis pathology. Several inflammatory mediators released by the synovium have been implicated in the regulation of pain, including S100A8 and S100A9 which may regulate pain either via direct stimulation of TLR4 on the nerve endings in the synovium or via stimulation at the site of the dorsal root ganglia (DRG), thereby enabling an increased phagocyte infiltration, which may cause sensitisation.

Objectives To elucidate the role of S100A9 in the pain response after induction of an acute synovitis using streptococcal cell walls (SCW) as a trigger, comparing S100A9-/- mice and their WT controls.

Methods Acute synovitis was induced by a single i.a. injection of SCW in the knee joint of C57Bl6 (WT) mice and S100A9-/- mice, control mice received an i.a. saline injection. Serum S100A8/A9 levels were investigated by ELISA. Joint swelling and cell influx was assessed by ${ }^{99} \mathrm{~m} \mathrm{Tc}$ accumulation and histology. Pain response were investigated using an Incapacitance Tester (weight bearing), Catwalk (gait analysis) and von Frey's filaments (mechanical allodynia). Gene expression of inflammatory mediators and neuron activation markers in DRG were determined by q-PCR. Monocyte influx and protein expression was monitored by immunohistochemistry (IHC).

Results A single i.a. injection of SCW resulted in increased synovial expression of S100A8 and S100A9 and subsequent increased serum S100A8/A9 levels at day 1, which returned to basal levels at day 7. Joint swelling and cell influx were similar in WT and $\mathrm{S} 100 \mathrm{~A}^{-/-}$mice at day 1 day excluding a role for S100A8/9 in the level of synovitis. WT mice showed a marked and significant decrease in percentage of weight bearing on the SCW injected hindpaw (28\%) compared to saline injection $(47 \%, \mathrm{p}<0.001)$ at day 1 , whereas $\mathrm{S}_{100 \mathrm{~A} 9^{-/-}}$mice did not. In addition, the stand-phase of the unaffected paws were significantly increased in WT mice 1 day p.i., while in

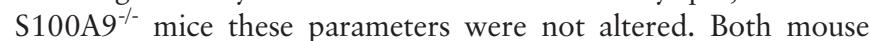
strains showed a similar reduction of paw withdrawal threshold, excluding a role for S100A8/9 in allodynia. Analysis of DRG showed no increased phagocyte infiltration after SCW injection and no change in gene expression of MCP-1, KC, IL-1 $\beta$ or TNF was measured. In addition, no change in F4/80 staining was seen in both WT and S100A9 ${ }^{-/}$mice. However, expression of neuron activation markers NAV1.7, ATF3 and GAP43 were significantly increased at 1 day after SCW injection in WT mice as compared to saline injected mice $(p=0.022,0.004$ and 0.030 , respectively) while no regulation was found in S100 A9 $9^{--}$mice, which is in line in with the reduced pain response observed earlier in $\mathrm{S} 100 \mathrm{~A}^{-/-}$mice. The difference in NAV1.7 expression in the DRG was further confirmed at protein level with IHC.

Conclusions These findings show that S100A9 is an important mediator of inflammatory nociceptive pain response in the knee, rather than being involved in peripheral sensitisation. During the acute phase of inflammation S100A8/A9 is likely regulated via direct activation of TLR4 on nerve endings in the synovium and not via monocyte infiltration in the DRG. Disclosure of interest None declared 